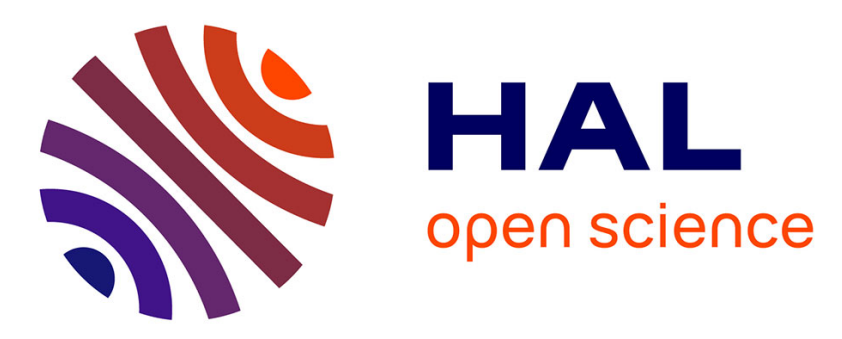

\title{
Evidence of Mechanically Activated Processes in Slow Granular Flows
}

\author{
K A Reddy, Yoel Forterre, O A Pouliquen
}

\section{To cite this version:}

K A Reddy, Yoel Forterre, O A Pouliquen. Evidence of Mechanically Activated Processes in Slow Granular Flows. Physical Review Letters, 2011, 106, 10.1103/PhysRevLett.106.108301 . hal01432106

\section{HAL Id: hal-01432106 https://hal.science/hal-01432106}

Submitted on 11 Jan 2017

HAL is a multi-disciplinary open access archive for the deposit and dissemination of scientific research documents, whether they are published or not. The documents may come from teaching and research institutions in France or abroad, or from public or private research centers.
L'archive ouverte pluridisciplinaire HAL, est destinée au dépôt et à la diffusion de documents scientifiques de niveau recherche, publiés ou non, émanant des établissements d'enseignement et de recherche français ou étrangers, des laboratoires publics ou privés. 


\title{
Evidence of Mechanically Activated Processes in Slow Granular Flows
}

\author{
K. A. Reddy, ${ }^{*}$ Y. Forterre, and O. Pouliquen ${ }^{\dagger}$ \\ IUSTI, CNRS UMR 6595, Université Aix Marseille, 5 rue Enrico Fermi, 13465 Marseille Cedex 13, France
}

(Received 7 December 2010; revised manuscript received 25 January 2011; published 11 March 2011)

\begin{abstract}
We study how a shear band in a granular medium dramatically changes the mechanical behavior of the material further in the non sheared region. To this end, we carry out a microrheology experiment, where a constant force $F$ is applied to a small rod immersed outside the shear band. In the absence of a shear band, a critical force $F_{c}$ is necessary to move the intruder. When a shear band exists, the intruder moves even for a force $F$ less than the critical force $F_{c}$. We systematically study how the creep velocity $V_{\text {creep }}$ of the rod varies with $F_{c}-F$ and with the distance to the shear band, and show that the behavior can be described by an Eyring-like activated process.
\end{abstract}

DOI: $10.1103 /$ PhysRevLett.106.108301

PACS numbers: 47.57.Gc, 83.80.Fg, 83.50.-v

Athermal systems encompass a wide variety of disordered soft materials such as foams, granular media, emulsions or colloidal suspensions. In all these materials, the media is composed of mesoscopic elements closely packed such that thermal fluctuations are negligible. As a result, these systems share peculiar flow behaviors intermediate between a solid and a liquid, such as the existence of a yield stress, shear banding [1-3], or finite size effects [4]. Recently, the idea that mechanical fluctuations in disordered athermal systems could be a source of noise analogous to temperature in thermal systems have motivated a large number of studies [1,5-12], in the spirit of the pioneering work of Bulatov and Argon [13] on the plasticity of amorphous solids. In this Letter, we experimentally address this picture in the context of granular flows. The rheology of granular flows in the dense regime has been extensively investigated over the past decade $[14,15]$. A phenomenological frictional viscoplastic constitutive law has been proposed, which captures many features of dense flows in the inertial regime far away from the flow threshold [16]. However, this simple approach, in which the local stresses are simply related to the local shear rate, does not account for important features close to the jamming transition, such as the development of quasistatic shear bands or the influence of the confinement when the system size becomes of the order of few tenths of particles. This failure of the local approach is likely related to cooperative effects, which have been documented in slow granular flows, such as correlations in the velocity fluctuations or in the force network [17-20]. How to capture these cooperative effects in a theoretical framework remains an open question and different approaches have been proposed. Some of them suggest that the mechanical noise induced by the flow itself could be an important source of non locality $[7,9,10]$. This picture seems to be supported by a recent experiment carried out in the Leiden group [21].

In this study, a steel ball resting on a bucket of sand was observed to sink as soon as a shear band was created at the bottom of the bucket, far away from the ball.
This observation suggests that the presence of a sheared region somewhere in the granular medium modifies the rheological properties of the sample everywhere. The study presented in this Letter is inspired by this striking experiment, but in a different geometry in order to study independently the role of the different parameters involved in the process, such as the distance to the shear band, the applied force and the stress state. The experiment is performed in a wide gap Couette cell, in which a shear band is created close to the inner cylinder. We then probe the rheology of the granular medium away from the shear band by trying to move a small rod immersed in the grains.
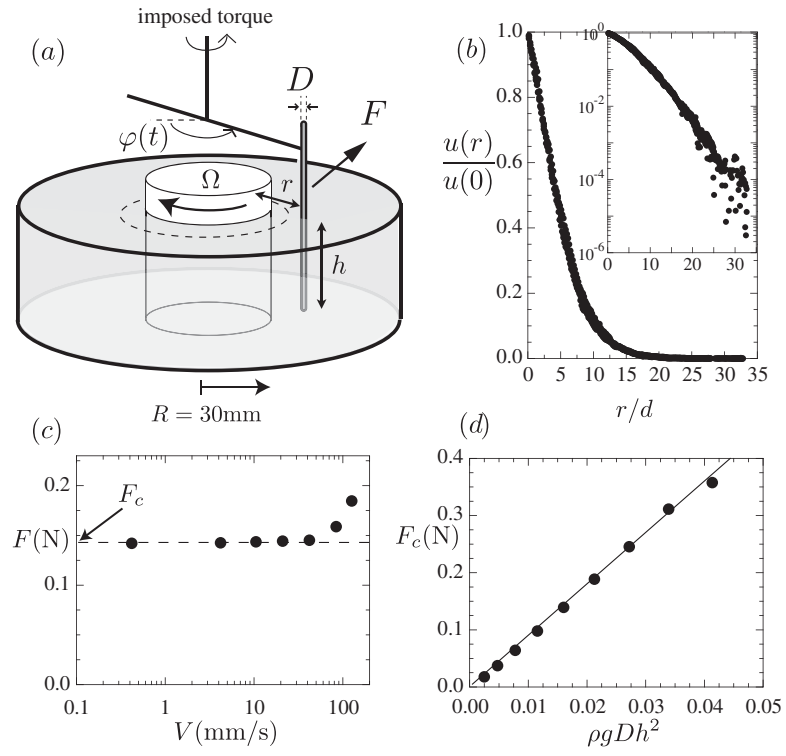

FIG. 1. (a) Experimental setup. (b) Shear band velocity profile normalized by the inner cylinder velocity $u(0)=R \Omega$ measured by Particle Image Velocimetry at the free surface. (c) Relation between the force and the velocity of the $\operatorname{rod}$ when $\Omega=0$ showing the existence of a critical force $F_{c}(h=40 \mathrm{~mm})$. (d) Critical force $F_{c}$ as a function of the rod depth $h$, showing a Coulomb-like scaling $\left(F_{c} \propto P S\right.$, where $P \propto \rho g h$ is the mean pressure and $S \propto h D$ is the rod surface). 
The experimental setup is presented in Fig. 1(a). The Couette device consists of a rotating inner cylinder (radius $R=30 \mathrm{~mm}$ ) made rough by gluing one layer of particles on it. The outer cylinder (radius $90 \mathrm{~mm}$ ) and the bottom are fixed and smooth. The gap is filled up to a depth of $70 \mathrm{~mm}$ of glass beads $d=0.5 \pm 0.05 \mathrm{~mm}$ in diameter. The inner cylinder rotates at a constant speed $\Omega$ by means of a belt connected to a motor [not drawn in Fig. 1(a)]. The rotation speed $\Omega$ is chosen less than $0.2 \mathrm{rad} / \mathrm{s}$ in order to stay in the quasistatic regime. The shape of the shear band is then independent of $\Omega$ and is shown in Fig. 1(b), where the velocity profile measured at the free surface is plotted. The grains velocity $u(r)$ decreases with the distance $r$ and presents an exponential tail over a distance of about 15 particle diameters, consistent with previous studies [14]. The purpose of the paper is to probe the rheology of the material in the creeping region, away from the shear band at a distance $r$ to the inner cylinder in the range $17 d<r<$ $55 \mathrm{~d}$. To do so, a long and thin $\operatorname{rod}$ of diameter $D=1 \mathrm{~mm}$ $(=2 d)$ is immersed into the material at a depth $h$ and at a distance $r$ from the inner wall. We then try to move the rod through the granular medium by imposing an orthoradial force $F$ thanks to a rheometer (Anton Paar MCR 501) [Fig. 1(a)]. Note that the force $F$ is always applied in the direction opposite to the inner cylinder rotation $\Omega$, as sketched in Fig. 1(a). We then precisely record the time evolution of the angular position of the rod $\varphi(t)$. One difficulty of the experiment is the long time evolution of the pile (compaction, structural aging). To minimize these effects and get reproducible results even for long experiments, we adopt the following protocol. Each run consists in two successive measurements, obtained for the same condition but with reversed sign for both the inner cylinder rotation and the direction of the force applied to the rod. This protocol, in which we change alternatively the direction of the flow, prevents the formation of a preferential structure and gives reproducible results.

We first study the motion of the rod when the inner cylinder of the Couette cell is not moving $\Omega=0$. In this case, a minimum force $F_{c}$ has to be applied in order to move the intruder. To precisely characterize this threshold, we impose a constant velocity $V$ and measure the drag force on the rod [Fig. 1(c)]. As long as the velocity is below $10 \mathrm{~mm} / \mathrm{s}$, the drag force is independent of velocity and independent of the position $r$ of the rod (in the range $17 d<$ $r<55 d$ studied). In the following, we define $F_{c}$ as the drag force for $V=4 \mathrm{~mm} / \mathrm{s}$ averaged over several turns. Figure 1(d) shows that $F_{c}$ scales as $\propto h^{2}$, compatible with a Coulomb-like friction for the drag force on the immersed rod [22].

We now fix the value of $F$ below $F_{c}\left(F / F_{c}=0.75\right)$ and turn to the case $\Omega \neq 0$, where a quasistatic shear band forms close to the inner cylinder. The rod is positioned in the "static" zone away from the shear band $(r=24 d)$. As soon as the inner cylinder is put in rotation, we observe that the rod is no longer stuck but starts to move forward in the direction of the applied force [Fig. 2(a) and the supplemental movie [23]]. This motion is all the more striking that it occurs in a region where no grain motion can be seen [the rod velocity is several orders of magnitude larger than the shear band velocity at this distance, Fig. 1(b)]. On short time scales, the motion of the rod is highly intermittent and consists of periods of slow creeping followed by rapid moves [Fig. 2(a), inset]. In this Letter, we do not address the fluctuating dynamics. Rather, we focus on the long time motion of the rod characterized by a well-defined constant creep velocity $V_{\text {creep }}=(R+r)\langle\dot{\varphi}\rangle$. In practice, the averaging time is chosen such that the total displacement of the rod is always larger than $50 D$, which can be as long as $30 \mathrm{~h}$ for the slowest creep measured. Figure 2(b) gives the creep velocity as function of the inner wall velocity $R \Omega$. In the range studied $(\Omega<0.2 \mathrm{rad} / \mathrm{s})$, the creep velocity of the rod is proportional to the wall velocity. This suggests that the creeping motion is controlled by quasistatic effects; $\Omega^{-1}$ being the only relevant time scale.

We now investigate how this creep velocity varies with the applied force $F$. Figure 3(a) gives the normalized velocity $V_{\text {creep }} / R \Omega$ as a function of the normalized force $F / F_{c}$ for a given distance $r=24 d$ and rod depth $h=$ $40 \mathrm{~mm}$. Data show that the creep velocity decreases over more than 4 decades as $F / F_{c}$ is varied from 0.9 to 0.5 . We also note that, even for $F / F_{c}=0.5$ corresponding to the slowest motion, the creep velocity of the rod is still at least 1 order of magnitude larger than the local grains velocity due to the shear band.

In Fig. 3(a), data are aligned in a semilog scale, meaning that the creep velocity depends exponentially upon $F / F_{c}$. This important result suggests that, as soon as the shear band is created, the rod moves for any applied force. In other words, the granular medium no longer exhibits a yield stress, as in the Leiden experiment [21]. We have checked that $F / F_{c}$ is the relevant control parameter by varying the rod depth $h$. Doing so, the threshold force changes as $F_{c}(h) \propto h^{2}$ according to Fig. 1(d). However, the creep velocity remains constant when the force $F$ is
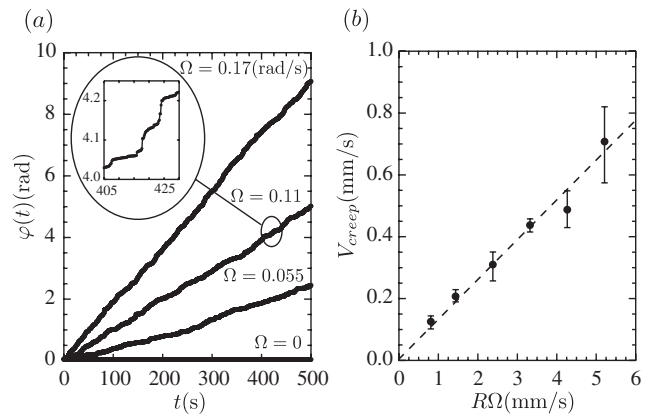

FIG. 2. (a) Rod angular displacement $\varphi(t)$ for different $\Omega$. (b) Mean creep velocity of the rod, $V_{\text {creep }}$, as a function of the inner wall speed $R \Omega\left(F / F_{c}=0.75, r=24 d\right)$. 

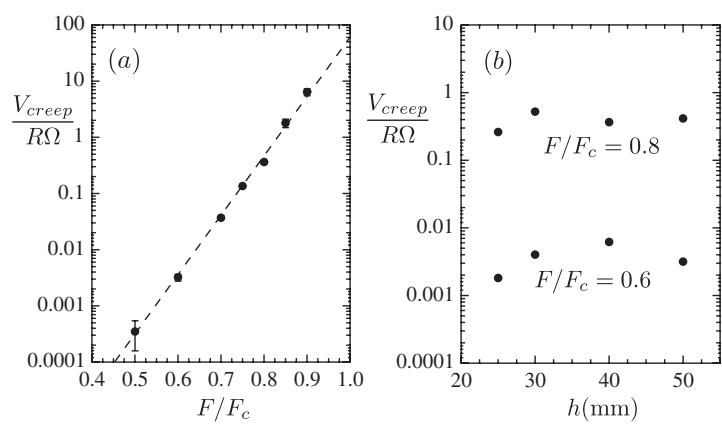

FIG. 3. (a) Normalized creep velocity of the $\operatorname{rod} V_{\text {creep }} / R \Omega$ as function of $F / F_{c}$ for $r=24 d$ and $h=40 \mathrm{~mm}$. (b) $V_{\text {creep }} / R \Omega$ as function of the rod depth $h$ for two values of $F / F_{c}$.

adjusted to keep the ratio $F / F_{c}(h)$ fixed [Fig. 3(b)]. All the previous results are obtained for a distance of the rod to the inner cylinder fixed $(r=24 d)$. Changing this distance strongly influences the creep velocity as shown in Fig. 4, which gives $V_{\text {creep }} / R \Omega$ as function of $F / F_{c}$ for different distances $18 d<r<42 d$. For each value of $r$, we recover the exponential decay of the creep velocity with distance to the threshold, the slope in lin-log becoming steeper and steeper as the rod moves away from the shear band. Notice that a small departure from an exponential is observed for the run close to the shear band $(r=18 d)$. An important observation is that all the data roughly converge to the same value $\left(V_{\text {creep }} / R \Omega \sim 63\right)$ when $F \rightarrow F_{c}$. Therefore, the creep velocity of the rod can be put in the form

$$
\frac{V_{\text {creep }}}{R \Omega}=A \exp \left[-\frac{F_{c}-F}{F_{c} G(r / d)}\right]
$$

where $A$ is a numerical constant equal to $A \sim 63$ and $G(r / d)$ a dimensionless function of the distance $r / d$. In order to estimate the function $G(r / d)$, we invert Eq. (1) and plot for each run $G=\left(1-F / F_{c}\right) / \ln \left(V_{\text {creep }} / A R \Omega\right)$ as a function of $r / d$. Figure 5 shows that $G(r / d)$ is a decreasing function of the radial distance, which extends up to 50

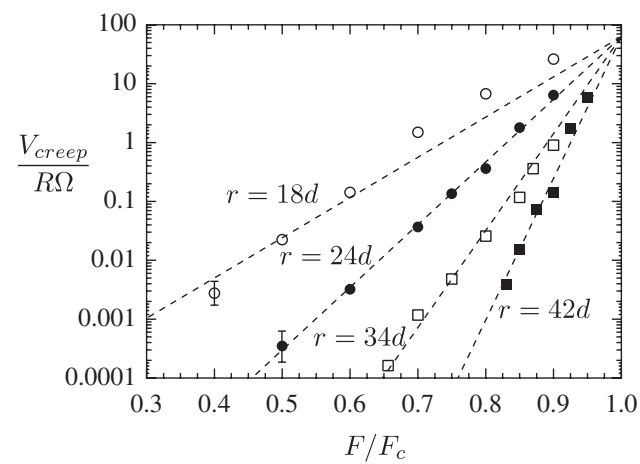

FIG. 4. $\quad V_{\text {creep }} / R \Omega$ as function of $F / F_{c}$ for different distances $r$ to the inner cylinder. The dotted lines are the best fit of the form (1) with $A=63$. particle diameters. A best fit with a power law gives $G(r / d) \sim(r / d)^{-1.45}$.

Equation (1) for the rod creep velocity can be interpreted in term of an "Eyring-like" activated process, by analogy with thermal systems [24]. Here, the analogue of the energy barrier is the distance to the critical force $\left(F_{c}-F\right)$ and the analogue of the temperature is $\delta F=F_{c} G(r / d)$, which can be interpreted as the magnitude of force fluctuations induced by the shear band. Force fluctuations scale with $F_{c}$ from dimensional arguments: in granular media made of rigid particles, the only stress scale is the confining pressure. Thus, force fluctuations on the rod have to scale with the integral of the hydrostatic pressure, which itself is proportional to $F_{c}$ [see Fig. 1(d)]. The function $G(r / d)$ describes the decay of the force fluctuation away from the shear band and could be interpreted as a stress propagator [25]. In this Eyring-like picture, the motion of the rod results from the force fluctuations send by the flowing shear band, which help the rod to overcome the force barrier with a probability proportional to a Boltzmann-like factor $\exp \left[-\left(F_{c}-F\right) / \delta F\right]$. The rate at which the fluctuations are sent (the attempt frequency in Eyring process) is given by the typical shear rate in the shear band, $R \Omega / d$. Finally, each time the rod crosses the barrier, it moves forward with a distance proportional to the grain diameter $A d$. This picture is compatible with a model of self-activated process induced by stress fluctuations proposed for the rheology of slow granular flows $[7,10]$, and more generally with plasticity models trying to take into account the self-induced mechanical noise in disordered athermal media $[1,6,9,12]$. However, interpreting the scaling observed in our experiments from a microscopic description remains an open challenge.

So far, all the results have been obtained for a given rod diameter $D=1 \mathrm{~mm}=2 d$. However, the large value of the numerical factor $A \sim 63$, which is connected to the distance of the jump in the Eyring picture, points to the role of the relevant length scale in our system. To address this issue, we measure the creep velocity of the rod for two

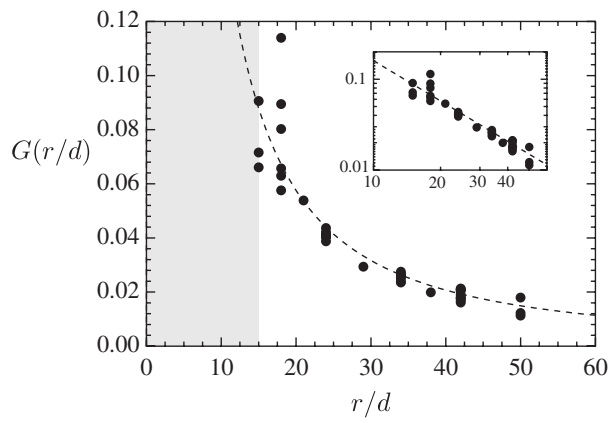

FIG. 5. Spatial range of stress fluctuations $G(r / d)$ measured for different values of $F / F_{c}$ as function of $r / d$ (inset in log-log scale). The grey zone gives the typical extension of the shear band ( $r<15 d$, see Fig. 1(b)]. The dotted line is the best power law fit of the data $G(r / d) \sim(r / d)^{-1.45}$. 


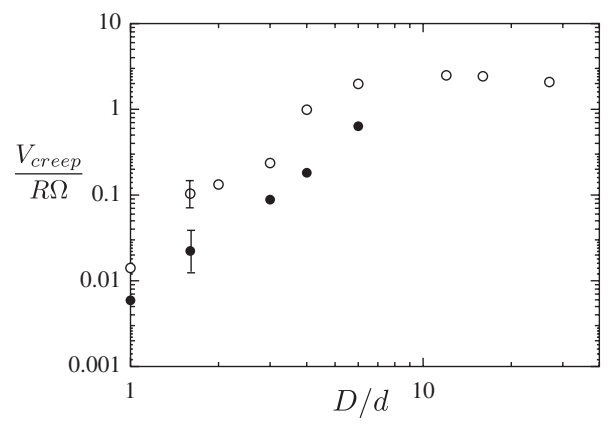

FIG. 6. Evolution of $V_{\text {creep }} / R \Omega$ as function of the normalized rod diameter $D / d$ for $F / F_{c}=0.85, r=24 d$ (white circle) and $F / F_{c}=0.7, r=34 d$ (black circle).

values of $F / F_{c}$ and $r / d$ for different rod diameters $(1<D / d<27)$. Figure 6 shows that the creep velocity actually strongly increases with $D / d$, before saturating at large values of $D / d$. This effect is not related to the rod elasticity, as checked by changing its stiffness while keeping its diameter fixed. The fact that thin rods move with more difficulty than large rods is reminiscent of the enhanced rigidity of small systems in granular media [15,26], and could be related to a typical length scale of the forcechain network in the pile. Another mechanism could be the self-interaction of the rod with its own wake, which becomes important for large rods.

In conclusion, our study suggests that stress fluctuations induced by a localized shear flow may help a granular medium to yield and overcome the flow threshold elsewhere. This self-activated process could play an important role in the rheology of dense granular flows close to the jamming transition, as it introduces cooperative effects not included in simple local approaches. Our measurements show that the rod velocity in the creeping region far away from the shear band scales exponentially with the distance to threshold $F_{c}-F$, even for the largest rods. This result contrasts with the linear velocity dependence of the force reported in the sinking ball experiment of the Leiden group [21]. However, in this experiment carried out in a bottomslip Couette geometry, the distance to the shear band and the confining pressure varied in an opposite manner during the sinking process, which makes the interpretation more complex. In our study, the exponential dependance of the velocity with the distance to threshold is a robust feature, which deserves to be checked in other configurations exhibiting shear banding such as heap flows [27,28] or channel flows [3-5]. This Eyring-like scaling represents a nontrivial test for theoretical approaches of slow granular flows, whether they derive from kinetic theory of granular media $[29,30]$ or from the rheology of soft glassy materials [12].

We thanks P. Nott for stimulating discussions, and funding from the Indo-French Centre for the Promotion of Advanced Research (IFCPAR Project No. 3404-1).
*Present address: Department of Physics, Clark University, Worcester MA, USA.

olivier.pouliquen@univ-provence.fr

[1] G. Debregeas, H. Tabuteau, and J. di Meglio, Phys. Rev. Lett. 87, 178305 (2001).

[2] L. Bocquet, W. losert, D. Schalk, T. Lubensky, and J. Gollub, Phys. Rev. E 65, 011307 (2001).

[3] L. Isa, R. Besseling and W. C. K. Poon, Phys. Rev. Lett. 98, 198305 (2007).

[4] J. Goyon, A. Colin, G. Ovarlez, A. Ajdari, and L. Bocquet, Nature (London) 454, 84 (2008).

[5] O. Pouliquen and R. Gutfraind, Phys. Rev. E 53, 552 (1996).

[6] P. Sollich, F. Lequeux, P. Hebraud, and M. Cates, Phys. Rev. Lett. 78, 2020 (1997).

[7] O. Pouliquen, Y. Forterre, and S. Ledizes, Adv. Compl. Syst. 4, 441 (2001).

[8] P. Schall, D. Weitz, and F. Spaepen, Science 318, 1895 (2007).

[9] R. P. Behringer, B. Dapeng, B. Chakraborty, S. Henkes, and R. Hartley, Phys. Rev. Lett. 101, 268301 (2008).

[10] O. Pouliquen and Y. Forterre, Phil. Trans. R. Soc. A 367, 5091 (2009).

[11] A. Lemaitre and C. Caroli, Phys. Rev. Lett. 103, 065501 (2009).

[12] L. Bocquet, A. Colin, and A. Ajdari, Phys. Rev. Lett. 103, 036001 (2009).

[13] V. Bulatov and A. Argon, Model. Simul. Mater. Sci. Eng. 2, 167 (1994).

[14] Gdr Midi, Eur. Phys. J. E 14, 341 (2004).

[15] Y. Forterre and O. Pouliquen, Annu. Rev. Fluid Mech. 40, 1 (2008).

[16] P. Jop, Y. Forterre, and O. Pouliquen, Nature (London) 441, 727 (2006).

[17] F. Radjai and S. Roux, Phys. Rev. Lett. 89, 064302 (2002).

[18] O. Pouliquen, Phys. Rev. Lett. 93, 248001 (2004).

[19] L. Staron, Phys. Rev. E 77, 051304 (2008).

[20] G. Lois, A. Lemaitre, and J. Carlson, Europhys. Lett. 76, 318 (2006).

[21] K. Nichol, A. Zanin, R. Bastien, E. Wandersman, and M. Van Hecke, Phys. Rev. Lett. 104, 078302 (2010).

[22] R. Albert, M. A. Pfeifer, A.L. Barabasi, and P. Schiffer, Phys. Rev. Lett. 82, 205 (1999).

[23] See supplemental material at http://link.aps.org/ supplemental/10.1103/PhysRevLett.106.108301.

[24] H. Eyring, J. Chem. Phys. 4, 283 (1936).

[25] G. Picard, A. Ajdari, F. Lequeux, and L. Bocquet, Eur. Phys. J. E 15, 371 (2004).

[26] M. Wyart, Europhys. Lett. 85, 24003 (2009).

[27] T. S. Komatsu, S. Inagasaki, N. Nakagawa, and S. Nasuno, Phys. Rev. Lett. 86, 1757 (2001).

[28] H. Katsuragi, A. R. Abatex and D. J. Durian, Soft Matter 6, 3023 (2010).

[29] J. T. Jenkins, Phys. Fluids 18, 103307 (2006).

[30] V. Kumaran, J. Fluid Mech. 599, 121 (2008). 